\title{
Phase Transition in a System of One-Dimensional Bosons with Strong Disorder
}

\author{
Ehud Altman, ${ }^{1}$ Yariv Kafri, ${ }^{1}$ Anatoli Polkovnikov, ${ }^{1}$ and Gil Refael ${ }^{2}$ \\ ${ }^{1}$ Department of Physics, Harvard University, Cambridge, Massachusetts 02138, USA \\ ${ }^{2}$ Kavli Institute of Theoretical Physics, University of California, Santa Barbara, California 93106, USA
}

(Received 6 February 2004; published 7 October 2004)

\begin{abstract}
We study one-dimensional disordered bosons at large commensurate filling. Using a real space renormalization group approach, we find a new random fixed point which controls a phase transition from a superfluid to an incompressible Mott glass. The transition can be tuned by changing the disorder distribution even with vanishing interactions. We derive the properties of the transition, which suggest that it is in the Kosterlitz-Thouless universality class.
\end{abstract}

DOI: 10.1103/PhysRevLett.93.150402

PACS numbers: 05.30.Jp, 64.60.Ak, 74.81.-g

Models of interacting bosons subject to disorder are important for understanding many real systems and present a considerable theoretical challenge. In particular, bosons in a disordered potential were studied extensively [1-7] in relation to granular superconductors, Josephson junction arrays, and ${ }^{4} \mathrm{He}$ on porous media. Very recently, there has been some interest in this problem in connection to experiments with ultra cold atoms $[8,9]$. These may provide a controllable environment for studying disorder and interactions.

Properties of granular superconductors and Josephson arrays are conventionally described using the $O(2)$ quantum rotor Hamiltonian

$$
H=-\frac{1}{2} \sum_{i} U_{i}\left(-\frac{\partial}{\partial \varphi_{i}}\right)^{2}-\sum_{i} J_{i} \cos \left(\varphi_{i+1}-\varphi_{i}\right),
$$

where $U_{i}$ is the grain charging energy and $J_{i}$ the Josephson coupling between grains. For uniform couplings, the zero temperature quantum partition function of the chain can be mapped to that of the twodimensional classical $x-y$ model with unit coupling and effective temperature $T^{\mathrm{eff}}=\sqrt{U / J}$ [10]. Thus, the pure system displays a superfluid-insulator transition of the Kosterlitz-Thouless (KT) universality class at a critical interaction strength $U \sim J$. In many real systems, such as granular superconductors, the couplings $U$ and $J$ are strongly disordered because of fluctuations in grain size and distance between grains. While weak disorder in $U$ and $J$ is perturbatively irrelevant, this does not rule out fixed points, and hence possible phase transitions, at finite disorder.

In this Letter, we identify a new quantum phase transition from a disordered superfluid to an incompressible gapless phase (Mott glass) using a real space renormalization group (RG). Remarkably, the fixed point that controls the transition corresponds to a classical model $\left(U_{i}=0\right)$. It can thus be tuned by varying the disorder in $J$ even at arbitrarily small charging energy $U$, in sharp contrast with the uniform case. We study the universal properties of the transition using the RG and show that it is characterized by exponential divergencies of length and time scales toward the transition. In addition to the RG treatment, we present a simple physical picture of the emerging phases and the phase transition consistent with the KT universality.

On passing, we note that the Hamiltonian (1) is equivalent to the Bose-Hubbard model at large commensurate filling [11], but it differs in some respects from the traditional dirty boson problem [1,3,5]. First, (1) obeys a local particle hole symmetry. Thus, it describes only the superfluid-insulator transition at commensurate filling. In addition, it does not contain the diagonal disorder $i v_{j} \partial / \partial \varphi_{j}$, which may become relevant for sufficiently strong interactions [1]. The significance of this difference was illuminated by recent Monte Carlo simulations of large two-dimensional systems, which considered both types of disorder [12]. While the diagonal disorder is expected to ultimately dominate, its effects may be unobservable at practical temperatures or length scales.

The real space RG we employ is originally due to Dasgupta and Ma [13]. It was successfully applied to study the universal properties of quantum spin chains $[14,15]$. In the spirit of Ref. [15], we define the cutoff energy scale $\Omega=\max _{i}\left\{U_{i}, J_{i}\right\}$. At each RG step, the element of the chain with largest energy is decimated, renormalizing the interaction constants nearby. Two types of steps are possible. If $\Omega=U_{i}$ for some site $i$, this site is assumed to be frozen in its lowest energy charge state. A connection between nearest neighbors of the decimated site is generated perturbatively:

$$
\tilde{J}_{i-1, i+1}=J_{i-1} J_{i} / \Omega \text {. }
$$

In the alternative case, when $\Omega=J_{i}$, the relative phase $\varphi_{i+1}-\varphi_{i}$ is assumed to be frozen in its ground state and the two sites are renormalized into a single one. The charging energy of the new site corresponds to additive recursion relations in terms of capacitances $C_{i} \equiv \Omega / U_{i}$ :

$$
\tilde{C}_{i}=C_{i}+C_{i+1},
$$

while the hopping connecting the new site with its neighbors remains unchanged. 
Repeated decimations gradually reduce the cutoff from its initial value $\Omega_{0}$ to a lower energy scale $\Omega$. Depending on the initial distributions of the couplings, two scenarios emerge: (i) Sites are joined together into ever growing superfluid clusters [see Fig. 1(a)] leading to a superfluid phase; (ii) A growing number of sites are eliminated to form disconnected clusters, leading to an insulating phase [see Fig. 1(b)].
It is convenient to describe the progression of the RG transformations and the cutoff energy scale with the parameter $\Gamma \equiv \log \left(\Omega_{0} / \Omega\right)$. In addition, we shall work with dimensionless coupling constants $\zeta_{i}=C_{i}-1$ and $\beta_{i}=\log \left(\Omega / J_{i}\right)$, characterized by probability distributions $f(\zeta, \Gamma)$ and $g(\beta, \Gamma)[16]$. Their flow with the decreasing energy scale is given by the master equations:

$$
\begin{aligned}
& \frac{\partial f(\zeta, \Gamma)}{\partial \Gamma}=(1+\zeta) \frac{\partial f(\zeta, \Gamma)}{\partial \zeta}+g_{0}(\Gamma) \int d \zeta_{1} d \zeta_{2} f\left(\zeta_{1}, \Gamma\right) f\left(\zeta_{2}, \Gamma\right) \delta\left(\zeta_{1}+\zeta_{2}+1-\zeta\right)+f(\zeta, \Gamma)\left(f_{0}(\Gamma)+1-g_{0}(\Gamma)\right), \\
& \frac{\partial g(\beta, \Gamma)}{\partial \Gamma}=\frac{\partial g(\beta, \Gamma)}{\partial \beta}+f_{0}(\Gamma) \int d \beta_{1} d \beta_{2} g\left(\beta_{1}, \Gamma\right) g\left(\beta_{2}, \Gamma\right) \delta\left(\beta_{1}+\beta_{2}-\beta\right)+g(\beta, \Gamma)\left(g_{0}(\Gamma)-f_{0}(\Gamma)\right)
\end{aligned}
$$

where $g_{0}(\Gamma) \equiv g(0, \Gamma)$ and $f_{0}(\Gamma) \equiv f(0, \Gamma)$. The first term in each of these equations describes the flow of the distribution due to redefinition of the cutoff following elimination of large couplings. The second term implements the recursion relations adding the renormalized coupling constants to the distribution. The last term removes the couplings neighboring the decimated ones and takes care of normalization of the distributions.

If either typical $\zeta \gg 1$ or $\beta \gg 1$, one can use $\delta\left(\zeta_{1}+\right.$ $\left.\zeta_{2}-\zeta\right)$ instead of $\delta\left(\zeta_{1}+\zeta_{2}+1-\zeta\right)$ in (4). Then the master equations are solved using the ansatz:

$$
f(\zeta, \Gamma)=f_{0}(\Gamma) \mathrm{e}^{-\zeta f_{0}(\Gamma)}, \quad g(\beta, \Gamma) e^{-\beta g 0(\Gamma)},
$$

where $f_{0}$ and $g_{0}$ obey:

$$
\begin{gathered}
\frac{d f_{0}(\Gamma)}{d \Gamma}=f_{0}(\Gamma)-f_{0}(\Gamma) g_{0}(\Gamma) \\
\frac{d g_{0}(\Gamma)}{d \Gamma}=-g_{0}(\Gamma) f_{0}(\Gamma)
\end{gathered}
$$

Thus,

$$
f_{0}(\Gamma)=g_{0}(\Gamma)-\ln g_{0}(\Gamma)-1+\epsilon .
$$

It is interesting to note that Eqs. (6) and (7) acquire the form of the KT flow equations, when written in terms of

(a)

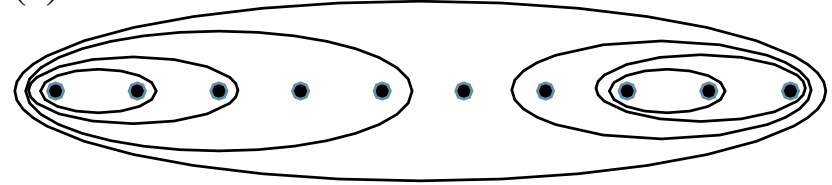

(b)

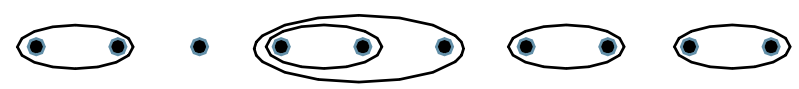

FIG. 1 (color online). Possible fates of the chain after repeated application of the RG rules. (a) Superfluid phase: sites are joined into ever growing clusters. (b) Insulating phase: clusters become effectively disconnected at low energy scales. $y=\sqrt{f_{0}}$ and $x=g_{0}$. The integration constant $\epsilon$ controls the flow as depicted in Fig. 2. Distributions with $\epsilon>0$ flow to $f_{0} \rightarrow \infty$ and $g_{0} \rightarrow 0$. This corresponds to vanishing Josephson coupling and $U \rightarrow \Omega$, namely, an insulating phase. For $\epsilon<0$ and $g_{0}>1$, the flow approaches a line of fixed points with a nonuniversal $g_{0}(\infty)=1+\alpha$, $\alpha>0$, and $f_{0}(\infty)=0$. This corresponds to an array of Josephson junctions with no charging energy and a power-law distribution of couplings $p(J) \propto(J / \Omega)^{\alpha}$. The critical flow occurs when $\epsilon=0$. It terminates at the unstable fixed point $g^{*}=g_{0}(\infty)=1(\alpha=0)$ and $f^{*}=$ $f_{0}(\infty)=0$. Note that even a system with vanishingly small charging energy and random tunneling can be tuned through the critical point by changing the disorder distribution. When $\alpha<0$, the system flows to the insulating phase.

We now apply Eqs. (6) and (7) together with the asymptotic forms of $g_{0}(\Gamma), f_{0}(\Gamma)$ to show that the transition has KT-like universal properties and dynamical exponent $z=$ 1. First, we establish a connection between energy and length scales. As the RG flow proceeds, more sites are eliminated or joined into larger superfluid clusters. Thus,

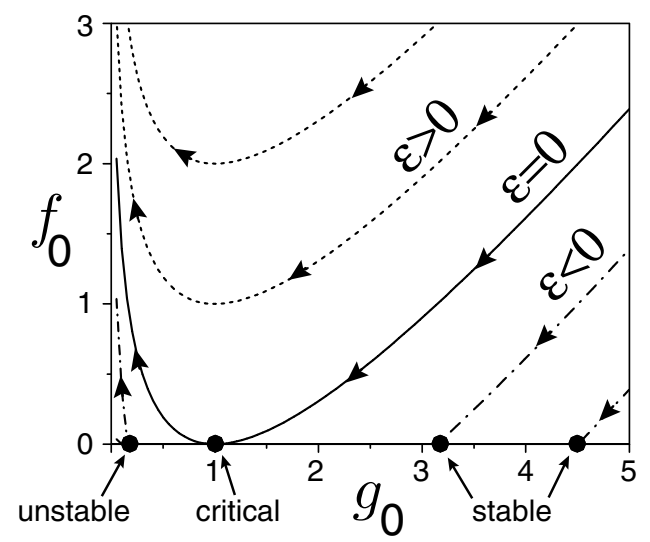

FIG. 2. Flow of the parameters $f_{0}(\Gamma)$ and $g_{0}(\Gamma)$. Lines of constant $\epsilon$ are plotted. The axis $f_{0} \equiv 0$ represents a stable fixed line for $g_{0}>1$ and unstable for $g_{0}<1$. 
the number of sites at scale $\Gamma$ decreases as

$$
N(\Gamma)=N_{0} e^{-\int_{0}^{\Gamma}\left(f_{0}+g_{0}\right) d \Gamma^{\prime}} .
$$

The length scale associated with $\Gamma$ is the size of clusters. In units of the original lattice constant, it is given by $l(\Gamma)=N_{0} / N(\Gamma)=\exp \left[\int_{0}^{\Gamma}\left(f_{0}+g_{0}\right) d \Gamma^{\prime}\right]$.

Solving the flow Eqs. (6) and (7) near the fixed point, $g_{0}^{*}=1, f_{0}^{*}=0$, we can identify typical time and length scales, which characterize the critical flow:

$$
\tau_{c} \propto e^{1 / \sqrt{\epsilon}}, \quad \xi_{c} \propto e^{1 / \sqrt{\epsilon}},
$$

where the exponential divergence as the tuning parameter $\epsilon \rightarrow 0$, suggests a transition in the KT universality class with a dynamical exponent $z=1$.

Using the universal distributions found above, we can calculate some observables. For example, to find the compressibility, we apply the RG to a finite chain down to the scale $\Gamma_{1}$ where only a single site is left $\left[N\left(\Gamma_{1}\right)=1\right]$. The compressibility extracted from the renormalized Hamiltonian of the remaining site $H_{1}=U n^{2}$ then reads:

$$
\kappa=\left\langle\frac{1}{N_{0} U}\right\rangle \approx \frac{1}{N_{0} \Omega\left(\Gamma_{1}\right)} \int_{0}^{\infty}(\zeta+1) f_{0}\left(\Gamma_{1}\right) e^{-f_{0}\left(\Gamma_{1}\right) \zeta} d \zeta
$$

Using Eqs. (9), (6), and (7) to derive the asymptotic forms of $f_{0}(\Gamma)$ and $g_{0}(\Gamma)$ we find in the insulating regime, $\kappa_{i n} \propto$ $\left(\ln N_{0}\right) / N_{0}$, which vanishes in the thermodynamic limit. Similarly in the superfluid side $\kappa_{s f}=k C_{0}$, where $k$ is a positive nonuniversal constant and $C_{0}=1 /\left[f_{0}(0) \Omega_{0}\right]$ is the average capacitance at the outset. It follows that $\kappa$ is discontinuous at the transition. In a similar way, we find the gap in the superfluid state obeys $\Delta \sim \ln N_{0} / N_{0}$. In the insulating phase, the gap vanishes as $\Delta \sim 1 / \ln N_{0}$. This is because of rare large clusters with arbitrarily small charging energy. Thus, we identify the insulating phase as a Mott glass, incompressible yet gapless, similar to the Mott glass found in a system with potential disorder [17].

We have implemented the RG scheme numerically to verify that the solution (5) is an attractor of the flow for generic initial disorder. We started from chains with $2.5 \times$ $10^{6}$ sites, and different forms of initial disorder distributions. The general features of the flow diagram appear to be insensitive to the type of distributions used. A specific example calculated from various box distributions of $J$ and $U$ is given in Fig. 3. The result clearly shows that the distributions converge to the solutions (5), demonstrating that they are stable attractors of the RG flow. The positions of the minima marked by a line in Fig. 3 agree with those predicted by the first correction to (6) from expansion of the $\delta$ function in Eq. (4) to the lowest order in $1 / \zeta$.

The real space RG is usually justified near infinite randomness fixed points $[14,15]$. Here, the validity of the recursion relations (2) and (3) relies on the distribution of $J$ 's being wide in the sense that $\langle J\rangle \ll \Omega$. Clearly

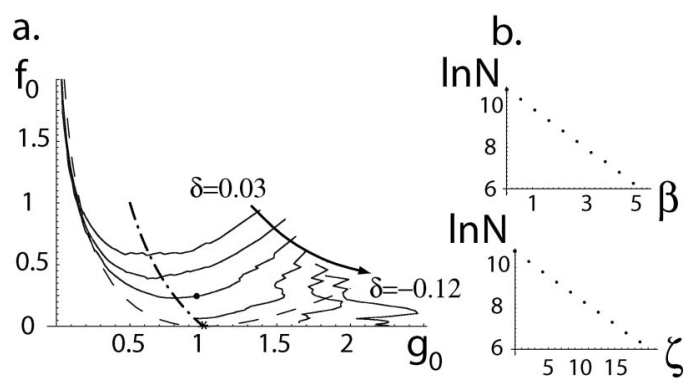

FIG. 3. Numerical RG flows for systems with initial box distributions. (a) Distribution of $U$ is centered around $0.7+$ $\delta / 2$ and that of $J$ around $0.7-\delta / 2$ with $\delta=$ $0.03,0,-0.03,-0.06,-0.09,-0.12$. The width of both distributions is always taken to be $1 . f_{0}$ and $g_{0}$ are defined as the inverse of the numerical average of $\zeta$ and $\beta$, respectively. The dashed line marks the superfluid-insulator separatrix according to Eq. (8) with $\epsilon=0$. (b) A semilog plot of the distributions of the couplings $\zeta=\Omega / U-1$ (top) and $\beta=\ln \Omega / J$ (bottom) at a typical point in the flow not far from the critical point [marked by a black dot in (a)].

this does not hold at the critical point where $\langle J\rangle=\Omega / 2$. While the relation (2) can be controlled by the smallness of $\langle U\rangle$ near the critical point, corrections to (3) produce next nearest neighbor Josephson couplings of order $\langle J\rangle^{2} / \Omega$. To justify the RG scheme, we need to show that longer range terms are irrelevant. The corresponding analysis is rather complicated and we defer it to a future work. Here, we rederive the phase transition and explain its nature using an alternative physical argument.

To understand the origin of the fixed point distributions, we employ two simple toy models (see also Ref. [7]). First, consider the insulating fixed point. We assume that the Josephson couplings take the values $J_{i}=$ 1 with probability $q$ and $J_{i}=0$ with probability $1-q$ and the charging $U_{i}=u \ll 1$ is uniform. Such a chain consists of disconnected clusters and thus can describe only the insulating phase. The cluster sizes are distributed according to $P(N)=q^{N}$, and the effective interaction is given by $U(N)=u / N$. From this, we immediately see that $f(\zeta)$ is indeed given by (5) with $f_{0}=|\ln q|$. Clearly, the insulating phase is stable towards weak Josephson couplings between the clusters. Therefore, the disconnected chain used in this toy model is fairly generic once we deal with an insulator.

We can describe the superfluid fixed line considering a different chain with uniform Josephson coupling $J_{i}=$ $b \ll 1$, and charging energies having a binary distribution: $U_{i}=1$ with probability $q, U_{i}=0$ with probability $1-q$. We may perturbatively eliminate the sites with $U_{i}=1$. The remaining neighboring sites are connected by new effective couplings $J=b^{N}$, where $N$ is the size of the eliminated block between them (see Fig. 4). In addition, the size distribution of such blocks is $P(N) \sim q^{N}$. The system is thus described by an effective classical chain of Josephson junctions (no charging energy) with a 


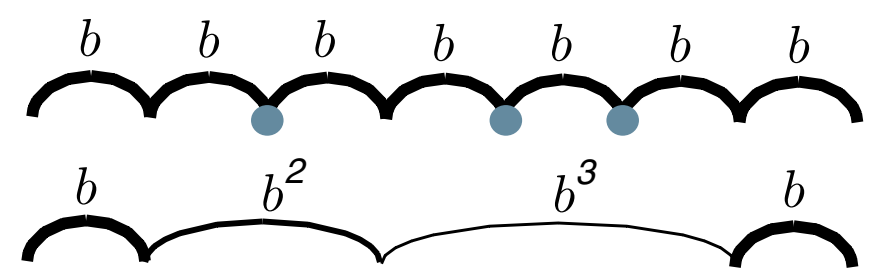

FIG. 4 (color online). Schematic representation of the second toy model. The circles on the top graph denote sites with a large charging energy $U=1$. After eliminating these sites we arrive to a classical chain with disordered couplings between the neighboring sites (bottom plot).

power-law distribution of couplings:

$$
P(J)=P(N) \frac{d N}{d J}=(\alpha+1)(J / b)^{\alpha},
$$

where $\alpha=|\ln q / \ln b|-1$.

This simple derivation suggests that the classical chain is a stable fixed line for all $\alpha$. However, this is special to the toy model with a finite fraction of grains having $U=$ 0 . We now show that a small $U$ perturbation will destabilize the line for $\alpha<0$. Physically this is due to the vanishing thermodynamic stiffness, which is defined as: $\rho(L)=L(E(\theta)-E(0)) / \theta^{2}$. For a given realization of a chain of length $L$ we get $\rho(L)=L /\left(\sum_{i} 1 / J_{i}\right)$. In the limit $L \rightarrow \infty$ the average over the chain appearing in the denominator can be replaced by an average over the distribution yielding $\rho(\infty)=\max \{0, \alpha /(\alpha+1)\}$. This result supports the identification of the phase with $\alpha>0$ as a superfluid, sustaining a finite thermodynamic stiffness. On the other hand, the states with vanishing stiffness at $\alpha \leq 0$ are expected to be unstable to adding weak interactions. To see this more explicitly, consider perturbing the system with a small uniform $U_{i}=\Delta_{0}$. We choose an energy scale $\Omega$ such that $\Delta_{0}<\Omega \ll \Omega_{0}=b$. The weak bonds with $J<\Omega$ are separated by clusters of average size $L=\left(\Omega_{0} / \Omega\right)^{\alpha+1}$ consisting only of strong bonds $(J \geq \Omega)$. Since each cluster is superfluid by the choice of parameters, the charging energy of a cluster of size $l$ is given by $\Delta(l) \sim \Delta_{0} / l$. Because clusters are independent from each other, the distribution of their sizes is simply $P(l)=L^{-1} \exp (-l / L)$. So, the average charging energy on the coarse grained lattice is $\Delta \sim \Delta_{0} \ln L / L$. Putting everything together we find:

$$
\frac{\Delta}{\Omega} \sim\left(\frac{\Delta_{0}}{\Omega_{0}}\right)\left(\frac{\Omega}{\Omega_{0}}\right)^{\alpha} \ln \left(\frac{\Omega_{0}}{\Omega}\right)=\left(\frac{\Delta_{0}}{\Omega_{0}}\right) \Gamma e^{-\alpha \Gamma} .
$$

Therefore, the charging energy is relevant for $\alpha \leq 0$ and irrelevant for $\alpha>0$, in agreement with the RG results.

The flow towards an insulator for $\alpha<0$ implies that the superfluid stiffness is suppressed exponentially with the system size at large $L$. On the critical point $\alpha=0^{+}$, on the other hand, $\rho(L) \sim 1 / \ln L$. This is because the smallest $J_{i}$ on a chain is of order $1 / L$ and serves as a lower cutoff for the average $\left\langle 1 / J_{i}\right\rangle$. For $\alpha>0$, the superfluid stiffness remains finite in the thermodynamic limit. The collective modes in the superfluid can be described by a harmonic chain with off diagonal disorder (see, e.g., Ref. [18]). In particular, it follows that all finite energy phonons in the disordered superfluid are localized [19].

In this Letter we studied a disordered 1D $O(2)$ quantum rotor model. Using real space RG analysis, we found a new random fixed point that controls a transition between an incompressible Mott glass and a superfluid phase. The new phase transition is consistent with KT universality, with a dynamical exponent $z=1$. Interestingly, the fixed point corresponds to a classical model with a vanishing charging energy and uniform distribution of Josephson couplings.

We are grateful to L. Balents, E. Demler, D. S. Fisher, M. P. A. Fisher, S. M. Girvin, D. Greenbaum, A. Paramekanti, N. Prokof'ev, S. Sachdev, and D-W. Wang for useful discussions and comments. This work was supported by ARO and NSF under Grants No. DMR0233773, No. DMR-0231631, No. DMR-0213805, No. DMR-0229243, and No. PHY99-07949.

[1] T. Giamarchi and H. J. Schulz, Europhys. Lett. 3, 1287 (1987); Phys. Rev. B 37, 325 (1988).

[2] M. Ma, B. I. Halperin, and P. A. Lee, Phys. Rev. B 34, 3136 (1986).

[3] M. P. A. Fisher, P. B. Weichman, G. Grinstein, and D. S. Fisher, Phys. Rev. B 40, 546 (1989).

[4] K. G. Singh and D. S. Rokhsar, Phys. Rev. B 46, 3002 (1992).

[5] J. K. Freericks and H. Monien, Phys. Rev. B 53, 2691 (1996).

[6] D. Dalidovich and P. Phillips, Phys. Rev. Lett. 89, 27001 (2002).

[7] N. Bray-Ali and J. E. Moore, Phys. Rev. B 69, 184505 (2004).

[8] B. Damski et al., Phys. Rev. Lett. 91, 080403 (2003).

[9] D-W. Wang, M. D. Lukin, and E. Demler, Phys. Rev. Lett. 92, 076802 (2004).

[10] S. L. Sondhi, S. M. Girvin, J. P. Carini, and D. Shahar, Rev. Mod. Phys. 69, 315 (1997).

[11] S. Sachdev, Quantum Phase Transitions (Cambridge University Press, Cambridge, England, 1999).

[12] N. Prokof'ev and B. Svistunov, cond-mat/0301205.

[13] C. Dasgupta and S. K. Ma, Phys. Rev. B 22, 1305 (1980).

[14] D. S. Fisher, Phys. Rev. B 50, 3799 (1994).

[15] D. S. Fisher, Phys. Rev. B 51, 6411 (1995).

[16] Given the joint distribution $F(\zeta, \beta, \Gamma), \quad f(\zeta, \Gamma)=$ $\int d \beta F(\zeta, \beta, \Gamma)$ and $g(\beta, \Gamma)=\int d \zeta F(\zeta, \beta, \Gamma)$.

[17] E. Orignac, T. Giamarchi, and P. Le Doussal, Phys. Rev. Lett. 83, 2378 (1999); T. Giamarchi, P. Le Doussal, and E. Orignac, Phys. Rev. B 64, 245119 (2001).

[18] Alexander et al., Rev. Mod. Phys. 53, 175 (1981).

[19] H. Matsuda and K. Ishii, Prog. Theor. Phys. Suppl. 45, 56 (1970). 